\title{
Mujer y política en Chile: los límites de la incidencia
}

\author{
María de los Ángeles Fernández Ramil ${ }^{1}$ \\ Fundación Chile XXI \\ mangeles.fernandez@chile21.cl
}

Casi al mismo tiempo que la opinión pública tomaba conocimiento de la disminución en tres puntos del porcentaje de candidatas a alcaldesas que competirá en las elecciones municipales de octubre de 2012, bajando de 17,5 en 2008 a 14,4\% en 2012, se difundía otra noticia tanto o más sombría para la situación de la ciudadanía política femenina en Chile. La Comisión de Gobierno del Senado no incorporó a la ley de primarias en discusión algún mecanismo que permita neutralizar el efecto diferencial de género que tendrá para las mujeres con aspiraciones políticas. La indicación del senador Fulvio Rossi, que tuvo el apoyo de importantes organizaciones feministas, en orden a "establecer un porcentaje mínimo de participación de candidatas en las elecciones primarias", fue desatendida. De esta forma, se sumará muy probablemente una elección más a la ya de por sí desventajosa carrera electoral que las mujeres enfrentan en Chile. Sobra la evidencia que demuestra que enfrentamos dificultades para acceder a recursos electorales decisivos como son las redes de contactos y el acceso al financiamiento. El descenso porcentual ya había sido anticipado, desde que se iniciara la administración del Presidente Piñera, con la incorporación de solo $18 \%$ de ministras al gabinete y por el $16 \%$ de candidatas en las primarias voluntarias y acotadas realizadas por la Concertación de Partidos por la Democracia para elegir a sus candidatos en 141 comunas a lo largo del país. No se logra revertir la tendencia instalada desde 1990 según la cual los hombres ocupan el 80\% de las listas de candidatos. 
El cruce de ambas noticias es sintomático de la situación que experimenta la participación política femenina, luego de haber tenido el primer gobierno de la historia de Chile conducido por una mujer entre 2006 y 2010. Hasta ese momento, se podía afirmar que la evolución de las mujeres en los espacios de decisión política había sido lenta, pero progresiva. Desde 1989 a la fecha, el porcentaje de senadoras ha aumentado de $2,6 \%$ a $13,1 \%$ y el de diputadas, de $5,8 \%$ a $14,2 \%$. El promedio de presencia parlamentaria femenina es de $13,9 \%$ al día de hoy, bajo todavía al compararlo con el promedio regional de $22,3 \%$. Más distantes estamos todavía del promedio que la OCDE ostenta en estos temas: $25 \%$. Los avances, con todo, han sido oscilantes. De $15,8 \%$ de parlamentarias en 2005 , se pasó a 14,2 en 2009. En el espacio local, la situación es similar. El porcentaje de concejalas en 2008 fue de $23 \%$ y de $12,5 \%$ en alcaldesas, siendo el primero menor que en 2004, donde se alcanzó 26,7\%. Si de cargos ministeriales se trata, la paridad de la era Bachelet posibilitó que las mujeres ocuparan la mitad de dichos cargos a nivel del ejecutivo. Su momento pick se produce en 2006, con el 50\% que representa 11 ministerios. A pesar de no haberlo sostenido permanentemente en los cinco cambios de gabinete de su administración, terminó con $45 \%$ de ministras. La comprensión restringida de la prensa acerca de su significado, remitiéndola a un fenómeno netamente matemático, llevó a que declara temprana -y equivocadamente- su fracaso.

Chile permanece anclado en una situación paradojal. Tuvo la primera mujer que llega a la presidencia por la vía de las urnas en un país importante de América Latina, la misma que hoy ostenta también por primera vez el cargo de subsecretaria general de Naciones Unidas a la cabeza de ONU-Mujer. Desde que ella dejara la presidencia, es cierto que se ha continuado la senda del protagonismo femenino, ahora en el ámbito social, que ha cobrado un protagonismo nuevo en el Chile post Concertación. Son los casos de Camila Vallejo, ex presidenta de la Federación de Estudiantes de la Universidad de Chile (FECH) y rostro de la movilización social más emblemática que se recuerde desde que se recuperara la democracia. A ello se suma la reciente elección de Bárbara Figueroa, primera mujer a la cabeza de la Central Única de Trabajadores (CUT). Pero tales casos no pasan de ser excepciones todavía o síntomas de una política de aparente integración (que en el mundo anglosajón 
denominan tokenismo), contrastando con la subrrepresentación política femenina en todas las instancias de decisión política. Las excepciones son útiles para que algunas voces recurran al argumento de que las mujeres avanzan y que es solamente cuestión de tiempo su llegada a las esferas decisorias. Tal situación se podría revertir sustantivamente si nuestro país dejara de mantenerse como una isla a la difundida práctica de las cuotas que se ha instalado en América Latina. Esta medida ha demostrado ampliamente su efectividad en el aumento de la presencia política femenina. 50 países del mundo han legislado sobre cuotas y 11 de ellos se encuentran en nuestro continente. Según datos de FLACSO al año 2005, los países con cuota habían logrado un aumento de presencia legislativa femenina de $9,5 \%$ frente a $2,9 \%$ de los que no la tienen. En el Senado, incluso, su impacto es mayor: $15,8 \%$ frente a $2,3 \%$. La evidencia de su éxito cuando son incorporadas a las legislaciones es abrumadora, y no solamente medido en incremento numérico. Está ampliamente documentada la influencia que han podido generar mujeres que acceden en condición de representantes de los puntos de vista específicos de grupos de mujeres o feministas ${ }^{2}$.

A la fecha, por donde se lo mire, persiste una evidente deuda con las mujeres en el terreno de la política electoral. En lugar de registrar la disminución de barreras para iniciar una carrera política, lo que vemos es su incremento. A la inexistencia de límites a los mandatos, se suma la ausencia de mecanismos de acción afirmativa, la persistencia de un sistema electoral binominal que se ha demostrado claramente lesivo para las oportunidades electorales femeninas y la falta de apoyos financieros en un contexto donde el tratamiento de este aspecto se inclina más por las exigencias de transparencia y menos por la equidad efectiva entre los candidatos. Si a ello añadimos que la responsabilidad por los asuntos domésticos sigue fuertemente anclada en la experiencia femenina, aspirar a desarrollar una carrera política supone una evidente "cuesta arriba". ¿Es muy exagerado afirmar, acaso, que las chilenas enfrentan el "peor de los mundos posibles"? Ante este cuadro, las organizaciones de mujeres no se han quedado de brazos cruzados. A la arremetida de Comunidad Mujer impulsando una campaña por la ley de cuotas (www.leydecuotas. cl), deben sumarse esfuerzos múltiples por entregar conocimientos, habilidades y destrezas a las candidatas que competieron en las 
elecciones municipales de octubre de 2012, entre los que destacan "Más mujeres el poder" (www.masmujeresalpoder.cl) y la Red Municipalista de Mujeres (www.iter.cl). Paralelamente, el SERNAM con el PNUD han lanzado el Programa "600 líderes políticas para Chile", en base al desarrollo de escuelas de formación de liderazgos en todo el país.

El diagnóstico anterior, además, debe situarse en un contexto que ha cambiado por cuanto el movimiento estudiantil del 2011 develó la crisis de representatividad del sistema político chileno. Los estudios de opinión revelan, sistemáticamente, el descenso de la credibilidad de instituciones como el Parlamento y los partidos así como de la distancia hacia las coaliciones políticas. La encuesta de la Corporación Humanas del año 2011 ya visibilizó que el ámbito político es uno donde las mujeres se sienten más discriminadas, en un $74 \%$, siendo solamente superado por la esfera laboral, en un $95 \%$. Ello contrasta, y puede ser fuente de frustraciones, porque el interés femenino por la política ha aumentado en 12 puntos desde la encuesta realizada en 2009. En cuanto a la evaluación de distintas instituciones políticas medulares, los partidos políticos reciben un $80 \%$ de desaprobación, luego del empresariado, los servicios públicos y el Congreso, con un $69 \%$. La negativa visión que se tiene de la institución parlamentaria se refuerza con los datos del sondeo CEP de agosto de 2012 donde las mujeres son las que revelan menor confianza.

El momento que atraviesa la ciudadanía política femenina, expresada no tanto en la capacidad de elegir sino de ser electa, levanta variadas preguntas en el marco del cambio de ciclo político que vive el país y donde la alternancia en el ejecutivo es solamente uno de sus indicadores. La primera, asentada en la idea de que la elección de la primera mujer Presidenta en la historia del país supuso un parteaguas para los asuntos relativos a la igualdad de género. Esta cobra, si cabe, más pertinencia ante la posibilidad de que la ex mandataria repostule al cargo en el marco de las elecciones presidenciales de 2013, dado su contundente posicionamiento en las encuestas de opinión. Los datos indicados acerca de la situación que atraviesa la participación política femenina abonan el escepticismo de primera hora que revelaron estudiosas de la realidad política de las mujeres en Chile. Tal es el caso de Franceschet, quien no 
disimuló su escepticismo acerca de la profundidad de los cambios que Bachelet podría acometer. La académica ya venía advirtiendo las dificultades existentes para que la representación numérica de las mujeres sirviese para impulsar la representación de los intereses de género, precisando que el conservadurismo moral y social de la clase política chilena, la propia debilidad del movimiento de mujeres con ausencia de vínculos entre sus diferentes segmentos y su escaso nexo con las mujeres que aspiran a cargos de representación popular junto con su propia heterogeneidad, configuraban un cúmulo de obstáculos que socavaban la relación entre representación numérica y sustantiva. A pesar de los cambios experimentados, la académica precisaba que existían ambigüedades en torno al rol de las mujeres como madres. Ello dificultaba las posibilidades de cambios efectivos en un gobierno de Bachelet ya que, para que acontecieran, tendrían que ser enfrentadas las tensiones entre el proceso de cambio cultural y su énfasis en la continuidad de las políticas económicas de Lagos ${ }^{4}$. A pesar de lo anterior, se señala que su mandato habría tenido un impacto simbólico. El estudio más emblemático en esta línea es el Informe de Desarrollo Humano ${ }^{5}$ del año 2010 para Chile, centrado en la igualdad de género. De esta forma, reseña "el efecto cultural de dicho acontecimiento, cuya característica más notoria ha sido la sensación de empoderamiento de las mujeres chilenas". Igualmente, recoge el impacto que habría tenido en las representaciones del liderazgo ya que, "a partir de ella la masculinización del liderazgo, que caracterizó a las primeras mujeres jefas de Estado del siglo pasado, dejó de ser la estrategia necesaria de legitimación para que una mujer pudiera ejercer un cargo de alta responsabilidad de manera eficaz". A ello se habría sumado "la instalación cultural en el imaginario de la ciudadanía del principio de paridad, a pesar de no haberse formalizado como una norma jurídica".

En el plano internacional, cuando Bachelet es analizada en comparación con mujeres que también han llegado a ocupar la primera magistratura, no se duda en señalar que "es la que más lejos habría llegado, tanto en su compromiso con el nombramiento de mujeres en el gabinete como en la promoción de políticas públicas en perspectiva de género" ${ }^{\prime \prime}$. Independientemente de los indicadores elaborados para medir su impacto, la promoción de la igualdad de género y su evaluación no es un asunto fácil. ¿Es posible afirmar 
que Bachelet ha ido más lejos, por ejemplo, que su par argentina, Cristina Fernández de Kichner? Aunque una respuesta movida por un primer impulso pudiera ser afirmativa, se requiere disponer de marcos comprensivos para evaluar, en perspectiva comparada, las políticas de igualdad de género. Htun y Laurel Weldon ${ }^{7}$ proponen una tipología que caracteriza las políticas que impactan o no los temas doctrinales y que, por otro lado, empoderan a las mujeres como grupo o bien abordan las desigualdades de clase. Desde esta perspectiva, la mandataria argentina parece haber ido más lejos que su par chilena, por ejemplo, en el tratamiento de temas que desafían la heterosexualidad normativa mientras la segunda parece haberse abocado más bien al tratamiento de la igualdad de género entre los sexos. Lo preocupante es que, con la presidenta chilena, se generaron un conjunto de condiciones más proclives para el logro de mayores avances, difíciles de replicar en un futuro inmediato. Díaz-Romero ${ }^{8}$ reconoce que si bien su presidencia "generó el contexto más fértil para la equidad de género, constituyendo un terreno propicio para el mayor despliegue discursivo en ámbitos previamente vetados como los derechos reproductivos y la participación política de las mujeres, no se concretaron medidas directamente orientadas a eliminar la discriminación y darle poder efectivo a las mujeres" (272).

Una segunda línea de interrogantes se relaciona con la situación del feminismo chileno post Bachelet. Autoras como Matamala ya anunciaban que "la igualdad de género podría terminar en cualquier parte, quedando a la intemperie en el nuevo escenario" y que "el sujeto político mujeres necesitará correr una maratón para neutralizar esta lúgubre escena con vestuario patriarcal confesional" (Díaz-Romero 165). Pese a este cuadro, un tanto desolador, la situación que atravesaría el movimiento de mujeres no concita total unanimidad entre las autoras. Mientras que Díaz-Romero (272) afirma que su fragmentación y desmovilización, unido a su desconexión con el gobierno (suspendida parcialmente durante el periodo de Bachelet por su propia red de relaciones con dicho mundo) explicaría, entre otros factores, la falta de avances simultáneos, sistémicos y globales en las distintas áreas que comprende la equidad de género, uno de los estudios más exhaustivos acerca del movimiento feminista conducido por Ríos, Godoy y Guerrero ${ }^{11}$ discute la tesis de su desmovilización. 
Argumenta que el feminismo no puede ser concebido como un actor único y homogéneo, sino como un campo de acción y que, en dicho marco, resulta más fructífero hablar de una transformación que tendió "a privilegiar estrategias de cabildeo e incidencia en agendas públicas por sobre el activismo político clásico". Las autoras, frente a las críticas que recibe la priorización de la estrategia de advocacy, precisan que ella emerge frente al decaimiento de otras formas de intervención en lo público, advirtiendo que la estrategia misma no debe ser vista como la causa del decaimiento de otras formas de accionar. En todo caso, lo que no se puede desconocer es que la opción del grueso del movimiento feminista por esta modalidad de acción supuso su incorporación en las estructuras y procedimientos existentes. En un artículo más reciente, Ríos y Mora ${ }^{12}$ reafirman su visión optimista, tanto de su situación como del accionar de los movimientos feministas en Chile, a pesar de la complejización del contexto político y social.

En todocaso, cabe preguntarse por los límites, las posibilidades y la pertinencia de dicha estrategia que sectores sustantivos del movimiento han seguido impulsando luego de la llegada al poder de la Coalición por el Cambio, de centro derecha, en marzo de 2010. La emergencia de un gobierno de orientación conservadora, junto con impulsar un enfoque de políticas públicas de género basado en el orden patriarcal tradicional, aunque ahora cubierto bajo un manto de aparente modernidad, no viene a representar solamente un cambio de ciclo para la política en su conjunto sino también para las demandas y aspiraciones de las mujeres. Para el movimiento feminista, además, supuso un cambio radical por cuanto anteriormente su accionar estuvo orientado hacia el Estado, colaborando estrechamente con el Servicio Nacional de la Mujer (Sernam). Las llamadas "femócratas", que fueron especialmente eficaces en la consecución de importantes avances gracias al despliegue de la llamada interfase múltiple vía institucionalidad cooperativa ${ }^{13}$ como forma de relación entre el Estado y la sociedad civil, han quedado literalmente huérfanas de interlocución frente a una nueva administración que ha prescindido de un importante contingente de profesionales con experiencia en igualdad de género formadas durante más de veinte años. Añádase a ello la desaparición del rol tradicionalmente activo que el ejecutivo jugó desde 1990 en el impulso de aspectos trascendentales de la 
agenda de género como en los casos de las leyes de divorcio y de filiación (Varas 177-178), aún dentro de los marcos estrechos que imponía la política de consensos y el veto político transversal (que se mantiene) a los asuntos considerados más polémicos como los relativos a los derechos sexuales y reproductivos y la participación política femenina.

El cambio de contexto, que incluye la posibilidad de que el gobierno conducido por Michelle Bachelet pueda ser interpretado como el fin de una etapa de la agenda de igualdad de género en la transición, demanda una profunda revisión de las estrategias utilizadas durante veinte años y sus alcances. Su fracaso en la tramitación reciente de la ley de primarias puede ser visto, bien como un hito más, o bien como un punto de inflexión, sobre todo si se sitúa en el marco de los llamados "núcleos duros" que frenan el avance hacia la igualdad señalados en el citado informe del PNUD (Varas 294-296). Es altamente probable que las feministas deban enfrentar ahora una nueva etapa de elusiones y evasivas, aunque bajo un ropaje argumentativo distinto al tradicional. Se recurrirá, muy seguramente, a las dificultades más imperiosas que enfrenta el sistema político para no dar curso a demandas problemáticas que, como las relativas a las cuotas, no solamente no concitan acuerdo en la clase política sino que son vistas como secundarias. El clima del debate público, por otra parte, se ha visto permeado por una cierta ideología maternalista que es posible desprender de los argumentos utilizados por algunos sectores para lograr la aprobación del postnatal de seis meses. Súmese a ello el hecho de que, "para seis de cada diez hombres, la igualdad de género se habría alcanzado"16, percepción que contribuye a relativizar la prioridad y urgencia que debiera otorgársele a la equidad de género. El sistema político chileno se encuentra hoy bajo una presión que, con intensidad espasmódica, se asienta en dos pilares: la crisis del sistema de representación, frente a la que la principal exigencia es el cambio del sistema binominal y los problemas de redistribución y desigualdad que no logran amortiguarse con políticas sociales compensatorias y frente a lo cual se ha erigido la demanda de una reforma tributaria. Arriagada ${ }^{17}$ sintetiza los indicadores del daño que experimenta nuestro sistema político cuando se refiere "al descrédito del gobierno y la oposición; del Parlamento, los partidos, la figura presidencial; 
la constante presencia de outsiders que expresa la búsqueda de un líder carismático que sacuda el statu quo; sobre todo, la incapacidad del sistema para procesar las demandas, las que van directamente a las calles y que no se canalizan ni en partidos ni instituciones".

Cabe preguntarse, entonces, por el sentido y la centralidad que el feminismo debiera darle al trabajo de incidencia en el marco de canales de intermediación que se encuentran en entredicho. No se trata de abandonar radicalmente dicha estrategia, cuya evaluación debe realizarse en el marco de otros factores como la estabilidad o inestabilidad de las alienaciones entre las elites, la presencia o ausencia de aliados influyentes y la capacidad y propensión gubernamental a la represión que, en el marco del actual gobierno, se ha demostrado que es mucha. Debiera preguntarse, también, por su heterogeneidad y evidente fragmentación. Respondidas estas preguntas, se sitúa un horizonte que hoy se observa menos imposible como es el cambio constitucional. Este pudiera ser un objetivo central y potencialmente orientador de la participación de las mujeres en lo que se visualiza como la posibilidad de una nueva etapa de democratización del país. Ya es posible advertir que las reformas políticas tradicionales y por largo tiempo postergadas, tales como el sistema binominal, que permitiría enfrentar el déficit de representatividad, o una nueva ley de partidos, pueden resultar insuficientes ante el cuadro que el ciclo de protestas estudiantiles, todavía en curso, ha generado. Frente a la estrategia gubernamental de actuación dentro de los límites del mercado, intensificando el rol regulador del Estado mediante el refuerzo de las superintendencias existentes o mediante la creación de otras nuevas, lo que parece ser el sustrato de las demandas que cruzan hoy al país es un cambio sustancial de derechos y obligaciones que interpela al Estado en el rol que estaría llamado a jugar en la provisión de servicios educativos. A ello se suma la demanda por transferencia de atribuciones y facultades relevantes a las regiones, asfixiadas por el hipercentralismo y que ya visibilizaron los movimientos en Magallanes y en Aysén, desde 2010 a la fecha. El impulso ciudadanizador por una nueva Constitución, por otro parte, provee una oportunidad para la convergencia de los distintos grupos y sectores agrupados en los llamados "feminismos" en torno a la movilización por un marco renovado y legitimado soberanamente de arreglos políticos que pueda acoger la noción, hasta hoy inexistente 
en nuestra legislación, de representación efectiva y de igualdad sustantiva. Frente a algo así, con toda seguridad, sería más difícil que se estrellase una ley de cuotas.

\section{Notas}

1. Directora Ejecutiva de la Fundación Chile 21, es cientista política de la Universidad Central de Venezuela, con Magister del Instituto de Ciencia Política de la Universidad Católica de Chile y estudios de Doctorado en Procesos Políticos Contemporáneos de la Universidad de Santiago de Compostela, España. Fue presidenta de la Asociación Chilena de Ciencia Política (2000-2002) e integró la Comisión Boeninger para la Reforma Electoral, en 2006. Escribe regularmente en distintos medios sobre la relación del género con la vida política, así como sobre reformas políticas. Sus áreas de interés alcanzan también a la calidad de la democracia y el rol de los partidos, el futuro de la socialdemocracia y la historiografía de la Ciencia Política. Su más reciente libro, del que es coeditora con Eugenio Rivera, se titula La trastienda del gobierno. El eslabón perdido en la modernización del Estado chileno (Catalonia, 2012). 2. Para más información sobre las variados intentos de una ley de cuotas en Chile, se sugiere consultar Fernández Ramil, María de los Ángeles Equidad política de género en el Chile actual: entre promesas y resistencias, Anuario de Derechos Humanos, Santiago, Universidad de Chile, 2008; Díaz-Romero, Pamela. "La mujer frente al poder". En La democracia frente al poder. Chile 1990-2010, de Augusto Varas, ed., Santiago: Catalonia, 2012, 254 y Comunidad Mujer Mujer y política: por una mayor participación femenina en la toma de decisiones públicas. No. 15, Santiago, 2012, 11.

3. Las chilenas siguen experimentando la condición de doble jornada de trabajo y segregación de género en el ámbito de lo público, a pesar de los cambios constatados luego de diez años en la encuesta Mujer, trabajo y familia realizada por el CEP en 2012. El cambio de percepciones no se acompaña de cambios conductuales en ámbitos como, por ejemplo, el reparto de tareas domésticas donde el 36\% de las mujeres considera que trabaja en tareas del hogar mucho más de lo que le corresponde frente al $31 \%$ de los hombres que cree que trabaja mucho menos de lo que debería.

4. Para más detalles, consultar Susan Franceschet. Women and politics in Chile. 2005, Boulder: Lynne Rienner Publishers y Susan Franceschet. “El triunfo de Bachelet y el ascenso político de las mujeres", Nueva Sociedad, 202: 13-22, 2008.

5. PNUD Desarrollo humano en Chile. Género: los desafíos de la igualdad 2010, Santiago. 6. Ma. de los Ángeles Fernández Ramil y Daniela Oliva Espinosa. Presidentas latinoamericanas e igualdad de género: un camino sinuoso. En Nueva Sociedad No. 240, 2012, 120.

7. En Mala Htun y S. Laurel Weldon. “When do gobernments promote women's rights? A framework for the comparative analysis of sex equality policy". Perspective on Politics 8 (1): 207-216, 2010.

8. Pamela Díaz-Romero. "La mujer frente al poder". En La democracia frente al poder. 
Chile 1990-2010.Augusto Varas, ed. Santiago: Catalonia, 2012.

11. Marcela Ríos, Lorena Godoy y Elizabeth Guerrero. ¿Un nuevo silencio feminista? La transformación de un movimiento social en el Chile postdictadura., Santiago: CEMEditorial Cuarto Propio, 2003.

12. Marcela Ríos y Claudia Mora. ¿De política de representación a política de coalición?: Posibilidades de movilización feminista en el Chile post-dictadura". Polis, 8 (24): 133-145, 2009.

13. Así la denomina Augusto Varas, que también hace referencia a la llamada trama institucional de equidad de género como forma de conexión y colaboración entre el Estado, los centros académicos independientes y universitarios y las ong's, posibilitando los avances en las políticas públicas en defensa de las mujeres. Para más detalles, consultar La reconstrucción democrática del espacio público. En La propuesta ciudadana. Una nueva relación sociedad civil-Estado. Santiago: Catalonia, 2006, 44-79.

16. Consultar Encuesta Images Chile. Resultados de la Encuesta Internacional sobre Masculinidades y Equidad de Género. Santiago, CulturaSalud-EmeMen+GenderEquality-UNFPA, 2011, 105.

17. Genaro Arriagada. ¿Vivimos una crisis política?, En La Tercera, 24 de junio de 2012. 